\title{
A comparison of HIV related advice in genitourinary medicine clinics with different histories
}

Vivian D Hope, Christine MacArthur

Objectives: To examine attendees reported experiences of health advice provision in genitourinary (GUM) clinics with different histories.

Design: A self-completed anonymous questionnaire was distributed at five clinics in the West Midlands Region of the United Kingdom.

Results: 297 of the 360 attendees approached returned completed questionnaires; $89.5 \%$ reported receiving health advice, $86 \cdot 4 \%$ found all of this easy to understand and $10 \cdot 4 \%$ wanted more advice. However, $33.9 \%$ received no advice on either HIV or safer sex. Those attending new clinics, set up since the HIV pandemic, were more likely than those attending older clinics, to have understood the advice given, to have had advice on both HIV and safer sex, and less likely to have wanted more advice. Among those attending with a concern about HIV, 14\% claimed to have received no advice on either HIV or safer sex; with no difference between old and new clinics. For those attending with reasons particularly relevant to receiving HIV related advice, but not with a concern about HIV, $40 \%$ claimed to have received neither HIV nor safer sex advice. In this sub-sample, those attending new clinics were more likely to have received advice on HIV as well as safer sex, and less likely to want more advice.

Conclusions: The results indicate that the provision of advice needs to be reconsidered, particularly in older clinics.

(Genitourin Med 1996;72:286-289)

Keywords: genitourinary medicine clinics; health advice

\section{Introduction}

In the United Kingdom one of the major consequences of the HIV pandemic has been the increased status and awareness of the genitourinary medicine (GUM) service. GUM clinics are not only the main sites for the diagnosis and treatment of sexually transmitted diseases, but are also involved in sexual health promotion. This later role developed from the contact tracing activities established when the incidence of gonorrhoea increased in the early 1960 's. ${ }^{1}$ Health promotion activities involve the provision of information, advice and counselling, either from specialist health advisors or other clinic staff, the arrival of HIV having markedly increased this. ${ }^{2}$

Concern was expressed by the mid 1980s about rising pressures on the GUM service resulting from HIV work. A Department of Health working party was set up to review this and found the service to be operating under intolerable conditions in many districts and generally ill-equipped to deal with demand. The report concluded that "GUM services must be designated as a priority" (p27), with additional resources made available. ${ }^{3}$

As a result the service developed in two ways. Firstly existing clinics, some of which were improved with more staff or better accommodation, have incorporated HIV into their established service provision. Secondly new clinics have been opened. These responses resulted in two types of clinics and it is plausible that their differing histories might affect the nature of HIV service provision. This paper investigates this possibility.

\section{Methods}

The study population was users of five GUM clinics within the West Midlands Region, sampled in the first six months of 1994. The clinics were chosen to represent a cross-section of those in the region. Inclusion was offered to all those attending during the sampling period, which took place on a number of days over a two week interval, to cover all time periods that each clinic was open. Subjects were approached in the waiting area by a research worker of the same gender and given a letter explaining the survey and stressing its anonymity and confidentiality. Those who agreed to take part were asked to self-complete the questionnaire during their visit and return it as they left. Help was provided to five individuals, who attended three of the clinics.

The questions covered demographic information, clinic accessibility, reason for attending, nature of the visit (first visit to this clinic, follow up or new episode) and health advice provision. Most questions had structured response categories. The attendees were not asked which category of staff had provided advice and definitions of safer-sex and HIV advice were not specified. The questions on health advice were answered by attendees at the end of their visit.

The five clinics were categorised into two
Address correspondence to: Dr V D Hope.

Accepted for publication

11 March 1996 
types based on their histories. The new clinics, were those established in response to the HIV pandemic. There were two of this type, both housed in spacious, well laid out, modern, purpose built accommodation. The old clinics, were those long established, pre-dating the HIV pandemic. Three clinics were of this type, housed in various forms of accommodation, but in all cases fairly cramped and poorly laid out.

The data was coded into a database. Chi square tests of association were carried out using SPSSPC+.

\section{Results}

Three hundred and sixty clinic attendees were approached and 297 completed questionnaires returned $(82.5 \%)$. Response rate did not differ between the clinic types or between gender of the attendees. A demographic summary of the sample is given in table 1 . Of the 297 attendees, $186(62.6 \%)$ were from the "Old" and $111(37 \cdot 4 \%)$ from the "New" clinic types. There were fewer who were in employment and more students at the old clinics $(60.5 \%$ employed compared with $78 \cdot 1 \%, p=0.0022$ ), but there were no other demographic differences between the two clinic types.

\section{Reasons for attending}

The attendees were asked "why are you attending the clinic today" and structured responses were provided. The most common reason for attendance was about genital warts $(35 \cdot 6 \%)$, followed by other sexually transmitted diseases (25.0\%); concern about HIV (15.8\%); a urinary problem $(9.9 \%)$; for hepatitis $B$ vaccination $(3.2 \%)$; and for some "other" reasons $(21 \cdot 1 \%)$. These "other" reasons which the respondents were asked to describe, included family planning, sexual health check-ups and cervical cytology, although in some cases a specific reason was not described. Some gave more than one reason for attending.

Table 1 Demographic characteristic of sample

\begin{tabular}{|c|c|}
\hline Characteristic & Number (\%) \\
\hline \multicolumn{2}{|l|}{ Sex: } \\
\hline $\begin{array}{l}\text { male } \\
\text { female }\end{array}$ & $\begin{array}{l}156(52 \cdot 5) \\
141(47 \cdot 5)\end{array}$ \\
\hline \multicolumn{2}{|l|}{ Age: } \\
\hline under 20 & $41(13 \cdot 8)$ \\
\hline $20-29$ & $151(50 \cdot 8)$ \\
\hline $30-39$ & $62(20.9)$ \\
\hline $40-49$ & $35(11 \cdot 8)$ \\
\hline 50 or over & $8(2 \cdot 7)$ \\
\hline \multicolumn{2}{|l|}{ Marital status: } \\
\hline single & $177(60 \cdot 0)$ \\
\hline married & $48(16 \cdot 3)$ \\
\hline living with partner & $36(12 \cdot 2)$ \\
\hline separated & $7(2 \cdot 4)$ \\
\hline \multicolumn{2}{|l|}{ How they fill their time: } \\
\hline working & $196(66 \cdot 4)$ \\
\hline seeking work & $29(9 \cdot 8)$ \\
\hline student & $32(10 \cdot 8)$ \\
\hline houseperson & $29(9 \cdot 8)$ \\
\hline non-worker & $7(2 \cdot 4)$ \\
\hline \multicolumn{2}{|l|}{ Sex of partner(s): } \\
\hline same sex/both sexes` & $19(6 \cdot 5)$ \\
\hline opposite sex & $275(93.5)$ \\
\hline \multicolumn{2}{|l|}{ Ethnic origin: } \\
\hline white & $259(87 \cdot 8)$ \\
\hline black & $24(8 \cdot 1)$ \\
\hline asian & $8(2 \cdot 7)$ \\
\hline other/mixed & $4(1 \cdot 7)$ \\
\hline
\end{tabular}

*Two males had partners of both sexes.
In view of the potential effects of the histories of the old and new clinics, comparison was focused on these two clinic types. The old and new clinics did not differ in the proportions attending for sexually transmitted diseases (STD's), hepatitis B vaccination, genital warts or urinary problems, but significantly more had attended the new clinics with a concern about HIV (22\% compared with $12 \%, \mathrm{p}=$ 0.0371 ) and more with some "other reason" ( $27 \cdot 6 \%$ compared with $17 \cdot 4 \%, \mathrm{p}=0 \cdot 0425)$. These differences may be due to variations in the range of services known to be provided, for example one clinic provided routine same-day HIV testing. Such variations will affect the clinic case mix. There were no differences between clinic types in terms of whether the attendee was making a first visit to the clinic, a follow-up visit or a return visit for a new problem.

\section{Advice}

Several questions were asked about advice and information received at the clinic. Only $10.5 \%$ of attendees reported receiving no information or advice during their visit. The respondents were asked "was the advice and information given easy to understand"; $86 \cdot 4 \%$ said it was; and "would you like to have been given more advice or information"; $10.4 \%$ said they would. When asked what kind of additional advice they wanted only 18 attendees responded, 11 of whom specified more advice on sexual health issues. Two specific advice questions were asked: "were you given any advice or information about HIVIAIDS" and "were you given any advice or information about safer sex"; $7 \cdot 5 \%$ reported advice only on HIV, $23.6 \%$ only on safer sex and $35.0 \%$ on both HIV and safer sex, while $33.9 \%$ reported advice on neither.

In the sample as a whole, significantly more attendees in the new compared with the old clinics reported receiving advice about HIV as well as safer sex $(51.0 \%$ and $25.6 \%, \mathrm{p}<$ 0.0001 ). In addition, significantly more in the new compared with the old clinics claimed to have understood all the advice received (94.8\% and $81.4 \%, p=0.0094)$; and fewer wanted more advice $(5.6 \%$ and $13.2 \%, \mathrm{p}=$ $0.0414)$. These differences, however, might simply reflect the known variations in case mix described above. If attending for family planning or cervical cytology, advice about HIV and safer sex would not be as appropriate. In addition, although safer sex and HIV advice at first attendance with a condition such as genital warts may be appropriate, several return visits are required for treatment when advice would not be necessary.

In order to consider this further, subgroups for whom advice on HIV and safer sex would be most appropriate were examined separately. Two sub-groups were studied: those attending because of a specific concern about HIV; and those attending for a reason particularly relevant to receiving advice about safer-sex or HIV (STDs, hepatitis B vaccination, warts other than treatment, or a urinary problem), but not with a specific HIV concern. 
Table 2 Comparison of the old and new clinic types: HIV and safer-sex advice among those attending with a problem particularly relevant to the provision of advice about safersex and HIV, but not with a specific concern about HIV ${ }^{\star}$

\begin{tabular}{llllll}
\hline \multicolumn{7}{c}{ Advice on Safer Sex and HIV } & & \\
\cline { 2 - 6 } Clinic type & $\begin{array}{l}\text { Advice on } \\
\text { neither (\%) }\end{array}$ & $\begin{array}{l}\text { Safer-sex } \\
\text { only (\%) }\end{array}$ & $\begin{array}{l}\text { HIV } \\
\text { only (\%) }\end{array}$ & $\begin{array}{l}\text { Advice on } \\
\text { both (\%) }\end{array}$ & Total \\
\hline Old clinics & $32(39 \cdot 0)$ & $30(36 \cdot 5)$ & $5(6 \cdot 1)$ & $15(18 \cdot 3)$ & 82 \\
New clinics & $13(41.9)$ & $2(6.5)$ & $3(9 \cdot 7)$ & $13(41.9)$ & 31 \\
Total & $45(39 \cdot 8)$ & $32(28 \cdot 3)$ & $8(7 \cdot 1)$ & $28(24 \cdot 8)$ & 113 \\
\hline
\end{tabular}

$\chi^{2}=12 \cdot 743 ; \mathrm{p}=0.0052$

Oor sexually transmitted diseases, hepatitis B vaccination, warts (other than for treatment) and/or a urinary problem.
Those attending with a specific concern about HIV

Forty five respondents $(15 \cdot 8 \%)$ reported attending with a specific HIV concern, and these were equally divided between the two clinic types (22 at old and 23 at new clinics). Six of these $(14 \%)$ claimed to have received no advice on either HIV or safer-sex, $21 \%$ advice only on HIV, $5 \%$ only on safer sex; and $60 \%$ on both. In this sub-group clinic types did not differ with regard to advice on safer sex or HIV; or whether attendees wanted more advice or information ( $9 \%$ said they would); or whether the advice and information was easy to understand ( $85 \%$ said it was). The clinics did not differ either in the proportion attending for the first time, for a follow up visits or returning with a new problem.

Those attending for a reason relevant to the provision of advice on HIV or safer sex, but not with a specific concern about HIV

One hundred and eighteen attendees (40\%) had not expressed a concern about HIV but had consulted with another reason relevant to the provision of advice about safer-sex or HIV. Within this sub-group attendees at the old clinics were more likely to report receiving advice only on safer sex, and those attending new clinic more likely to report receiving advice on both safer-sex and HIV. Similar proportions in both clinic types received no advice (40\%) (table 2 ). In addition, those attending old clinics were more likely than the new clinic attendees to have wanted more advice or information (table 3). The attendees understanding of the advice received however did not differ between clinic types ( $82 \%$ said it was easy to understand), nor did the nature of the attendees visits or their reasons for attending.

Table 3 Comparison of the old and new clinic groups by whether attendees wanted more advice: among those attending with a problem that is particularly relevant to receiving advice about safer-sex and HIV, but not with a specific concern about HIV ${ }^{\star}$

\begin{tabular}{|c|c|c|c|}
\hline \multirow[b]{2}{*}{ Clinic type } & \multicolumn{2}{|c|}{ Wanted more advice } & \multirow[b]{2}{*}{ Total } \\
\hline & No (\%) & Yes (\%) & \\
\hline $\begin{array}{l}\text { Old clinics } \\
\text { New clinics } \\
\text { Total }\end{array}$ & $\begin{array}{r}69(80 \cdot 2) \\
31(96 \cdot 9) \\
100(84 \cdot 7)\end{array}$ & $\begin{array}{c}17(19 \cdot 8) \\
1(3 \cdot 1) \\
18(15 \cdot 3)\end{array}$ & $\begin{array}{r}86 \\
32 \\
118\end{array}$ \\
\hline
\end{tabular}

\section{Discussion}

This survey of 297 GUM clinic attendees has produced encouraging results with regard to the provision of general advice and information, almost all receiving this, finding it easy to understand, and very few wanting any more.

Looking specifically at the provision of advice and information about HIV and safer sex however, a third of the sample reported nothing on either of these. This seemed high, although comparable with other work. In a study of four GUM clinics in England, Pillaye ${ }^{4}$ found that $54 \%$ of attendees reported HIV not being discussed during their visit, similar to the $57.5 \%$ who received no HIV advice in this study. Only $5 \%$ of the respondents in the study by Pillaye ${ }^{4}$ said they would have been offended had HIV been raised. The particular reason for the clinic visit in some cases might mean that the provision of advice on HIV and safer-sex is less appropriate, such as if returning several times for treatment, or attendance for a routine cervical smear. However, after removing these groups, apart from those attending with a concern about HIV/AIDS, the proportion receiving no advice or information on HIV or safer sex in this study remained substantially unchanged.

These findings are based on attendees reports of advice, so report bias should be considered. Attendees could purposefully misreport information, but the stress placed on the anonymity and independence of the study gave no reason for this. They may have had advice or information but not recognised it as such, or may not have wanted to see it as appropriate, thus disregarding it. If the latter, then health advising in the clinics has anyway been ineffective. Alternatively the doctor or health advisor may have ascertained that a particular individual was already well informed with further advice perceived as unnecessary. Even so it would still seem prudent to reinforce existing knowledge, address gaps, identify other issues adversely affecting risk behaviour and support positive behaviour changes. Pillaye ${ }^{4}$ noted that some gay men (no proportion given) who had received advice on safer sex from GUM clinics felt this to have been inadequate.

In this present study, among attendees who had visited the clinic because of a concern about HIV, $14 \%$ claimed to have received no advice or information about HIV or safer sex. Although this is much lower than in the rest of the sample it remains worrying since this subgroup were specifically seeking this. Their reason for attending described to us may not of course have been articulated to the clinic staff, presenting them with an entirely different reason. The two clinic types did not differ in the receipt of advice and information on HIV and safer sex for this sub-group of attendees, suggesting that clinic history has no effect here. For those requesting an HIV antibody test there is a standard procedure of providing pretest counselling, which might reflect more consistent practice in response to any expressed HIV concern.

Among the group attending with a reason 
relevant to receiving advice about safer sex and HIV, but not with a specific concern about HIV, the nature of advice reported in the old and new clinics did differ. More old clinic attendees reported advice about safer sex only, whilst both safer sex and HIV advice were more often reported at the new clinics. This could be due to different interpretations of advice given, or differences in the way this was presented in the clinics-older clinics might place more emphasis generally on safer sex, whilst newer clinics might relate advice more specifically to HIV.

Differences between old established clinics and those opened in response to the HIV pandemic, however, are plausible. HIV will have been incorporated into the procedures of the new clinics from their inception with differences in health promotion practice. Differences in environment might also have an affect. A Department of Health working party reported that some clinics operated in poor physical environments ${ }^{3}$ and a major report in 1993 highlighted difficulties with staffing levels and complements. ${ }^{2}$ The efficiency and effectiveness of the delivery of health advice could be adversely affected by these factors. For example, there may be insufficient staff to allow all attendees to see a health advisor or to allow time for other staff to provide advice, or a lack of a suitable space for health advising.

Two of the old clinics in this study were housed in adapted accommodation and all three were cramped, while both of the new clinics had spacious and well designed accommodation providing a more suitable environment for both staff and patients. At the new clinics time constraints on the staff generally seemed less and shorter waiting times for appointments were noted. One of the older clinics had no health advisor post, this role being undertaken by other staff and another had a post vacant; however, one of the new clinics also had no full-time health advisor as they were awaiting a new member of staff. As has been noted in previous studies cover for absent staff was also a problem. ${ }^{2}$ These possible effects of staffing and environmental factors on health advice needs further investigation.

Allen and $\mathrm{Hogg}^{2}$ noted the lack of emphasis placed on health education in some clinics and suggested that the balance between "education" and "counselling" needs reconsideration. They observed this to be a particular problem in two types of clinic: those with a traditional treatment and contact tracing approach, and those with a large amount of HIV related work. The demands associated with HIV testing and the care of those with
HIV antibody positive diagnoses have, in some clinics, been shown to take up most of the health advising time, thus limiting health education for other attendees. ${ }^{2}$

The findings of this study relate to attendees in the West Midlands Region of the UK, a region which has a population generally representative of the UK as a whole. The five clinics included represent approximately $20 \%$ of the GUM clinics in the region and were selected to reflect regional diversity. There were no indications that those attending during the study period were other than typical for the clinics included. The findings may not, however, be applicable to Greater London owing to the latter's higher HIV prevalence and reportedly large population of gay and bisexual men. ${ }^{6}$

Genitourinary medicine clinics access populations at risk of sexually transmitted diseases, thus with a corresponding elevated risk of HIV infection. ${ }^{5}$ Those who have concerns about their sexual well-being may also make use of the service. The national survey of Sexual Lifestyles and Attitudes found that individuals with large numbers of sexual partners were more likely to attend GUM clinics. ${ }^{6}$ As such these are clearly important sites for targeting sexual health messages.

The evidence from this present study raises a number of concerns about health education activities in GUM clinics. One third of the attendees claimed not to have received any advice or information on HIV or safer-sex during their visit and variations in the type of health advice reported was found between clinics with different histories. The reasons for these findings are important and need further investigation, focusing on clinic policy and its relation with practice.

This work has been funded by West Midlands Regional Health Authority. We thank those who took part in the survey and the clinics for allowing us access to their patients.

1 Thin RN. Health advisors (contact tracers) in sexually transmitted diseases. $B r \mathcal{F}$ of Venereal Dis 1984;60: 267-72.

2 Allen I, Hogg D. Work Roles and Responsibilities in Genitourinary Medicine Clinics London: Policy Studies Institute, 1993

3 Department of Health. Report of the Working Group to Examine Workloads in Genitourinary Medicine Clinics (The Monks Report). London: Department of Health, 1988.

4 Pillaye J. Preliminary results from a short patient survey of sexual health promotion in genitourinary medicine clinics. In: Pillaye, J ed. Sexual Health Promotion in ics. In: Pillaye, J ed. Sexual Health Promotion in Genitour 13 .

5 Quinn TC, Glasser D, Cannon RO, et al. Human immunodeficiency virus infection among patients attending clinics for sexually transmitted diseases. $N$ Eng $\mathcal{F}$ Med 1988; 318:197-203.

6 Johnson AM, Wadsworth J, Wellings K, Field J. Sexual Attitudes and Lifestyles. Oxford: Blackwell Scientific Publications, 1994. 